\title{
Scanning tunneling microscopy on rough surfaces-quantitative image analysis
}

\author{
G. Reiss, H. Brückl, J. Vancea, R. Lecheler, and E. Hastreiter \\ Institut für Angewaindte Physik III, Universität Regensburg, Universitätsstrasse 31, D-8400 Regensburg, \\ Germany
}

(Received 22 October 1990; accepted for publication 25 March 1991)

In this communication, the application of scanning tunneling microscopy (STM) for a quantitative evaluation of roughnesses and mean island sizes of polycrystalline thin films is discussed. Provided strong conditions concerning the resolution are satisfied, the results are in good agreement with standard techniques as, for example, transmission electron microscopy. Owing to its high resolution, STM can supply a better characterization of surfaces than established methods, especially concerning the roughness. Microscopic interpretations of surface dependent physical properties thus can be considerably improved by a quantitative analysis of STM images.

Many physical properties of solids are strongly influenced by the limiting surfaces. The thickness dependent resistivity of thin films, ${ }^{1-4}$ for example, can be understood only by taking into account the film's roughness. ${ }^{5-7}$ Typical roughnesses of polycrystalline surfaces, however, range between some $\AA$ and some $n m$ and usually cannot be directly observed except using scanning tunneling microscopy (STM). For surface dependent physical properties, a quantitative analysis of STM results thus is of great interest.

The preconditions for this analysis are reproducibility and reasonable resolution. In Refs. 8-10, we treated two main problems related with STM imaging of rough surfaces: resolution ${ }^{9}$ and deconvolution of images to real surface structures. ${ }^{10}$ Related problems have been discussed by other authors, too. ${ }^{11-16}$ Whereas reproducibility can be simply achieved using common STM designs, the resolution is a crucial point especially concerning the evaluation of roughnesses. ${ }^{9-16}$ This has been discussed in Ref. 10: using the STM image and a properly evaluated tip shape, those parts of the real surface can be calculated which had been in tunneling contact with the tip during scanning; concerning the remaining parts, appearing as "blackholes" in the calculated surface, no conclusions can be made except that they cannot be "seen" by the tip. In order to quantify the relation between the relative amount of black holes $A_{b}=F_{b l h .} / F_{S u r f}$ and the vertical, i.e., depthresolution $D_{r}=H_{\mathrm{STM}} / H_{\text {Surf }}$, Fig. 1 shows the calculated dependence of $D_{r}$ on $A_{b}$. Here, the surface has been assumed to consist of semi-elliptical islands with height $H_{\text {Surf. }}$ and lateral extension $a \cdot H_{\text {Surf. }}$. As Fig. 1 shows, $D_{r}$ strongly decreases with increasing $A_{b}$. Realistic values of the surface parameter are $3<a<8$. $A_{b}$ therefore should not exceed about $5 \%$ in order to obtain a reasonable depth resolution. Experimentally, values as large as $A_{b} \approx 0.2$ occurred by using blunt tips on polycrystalline $\mathrm{Ni}$ surfaces. Scaled to the largest depth found on the same sample with sharp tips $\left(A_{b}<0.03\right), D_{r} \approx 0.6$ can be experimentally estimated for this example in good agreement with Fig. 1.

STM was performed under ambient conditions with a typical tunneling resistance of 25-100 M $\Omega$ (see Refs.
8-10). The Ni films where partly covered with $1.5 \mathrm{~nm} \mathrm{Au}$ in order to evaluate the influence of oxidation. There, however, occurred no remarkable difference between the pure $\mathrm{Ni}$ surface and the samples protected by $\mathrm{Au}$. Here, mechanically prepared Pt tips have been used; whereas chemically etched $W$ tips often were damaged before tunneling occurred (probably due to tip-bending ${ }^{17,18}$ ), the Pt tips produced stable tunneling currents immediately. The amount of black holes shown by the STM images usually was smaller than $3 \%$, pointing to tip radii between 2 and 3

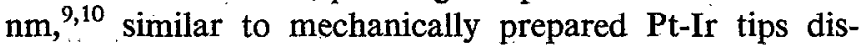
cussed in Ref. 18.

In order to obtain comparable results, we applied the following formalism for the quantitative evaluation of the STM topographies: Consider an STM image given by i $(x, y)(x, y$ : in-plane coordinates). The roughness can be described by the function $\mathbf{r}(h) d h$ defined as the ratio of those areas of $\mathbf{i}(x, y)$ at heights between $h$ and $h+d h$ and the whole surface. The function characterizes the distribution of roughnesses on the investigated surface. Concerning thin films, the mean surface height $h_{0}$ is additionally of interest. Using $\mathbf{r}(h) d h$ defined above, this can be found by stating, that volumes present above $h_{0}$ must be missing below. Thus, independent from the choice of $h=0$ before evaluating $\mathbf{r}(h) d h$, the correct value can be found by

$$
h_{0}:=\int_{-\infty}^{+\infty} h \cdot r(h) d h
$$

After shifting the height axis by $h_{0}$, the function $\mathbf{r}(h) d h$ represents the correct distribution of the surface roughness above $(h>0)$ and below $(h<0)$ the mean surface height. For flat substrates, $h_{0}$ can be identified with the mean film thickness.

The evaluation of island sizes usually turns out to be more difficult. This is due to the irregular shape of the islands which considerably influences results obtained, for example, from Fourier transforms of the STM image. A function very sensitive to typical lateral structures is the autocorrelation $\operatorname{acf}(\mathrm{a})$ defined by 


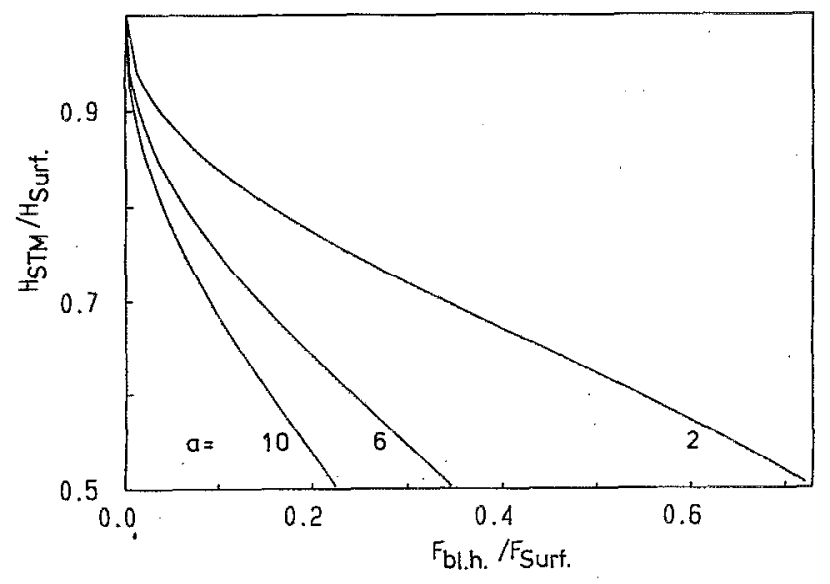

FIG. 1. The dependence of the relative depth resolution $H_{\text {STM }} / H_{\text {Surf. }}$ on the relative amount of black-hole surface $F_{b l . h} / F_{S u r f .}$ for a surface consisting of semi-elliptical islands. The parameter a is the lateral diameter of the islands scaled to their height.

$$
\operatorname{acf}(\mathbf{a}):=\frac{1}{S} \cdot \int_{S} \mathbf{i}(\mathbf{r}) \cdot \mathbf{i}(\mathbf{r}+\mathbf{a}) d^{2} \mathbf{r}
$$

with $\mathbf{r}, \mathbf{a}$ : in-plane coordinates; acf(a) shows a maximum at $\mathbf{a}=0$ equal to the square of the rms roughness. If the distribution of the island sizes follows a white noise law, the acf's exponentially decrease with increasing a. Additional features are caused by the specific distribution of island sizes. The location of these features, however, can be identified with the mean island diameter only for well defined lateral sizes. Realistic distributions, however, tend to smear out these structures of the acf's and to shift their locations to larger or smaller values depending on the unsymmetry of the distribution of the island sizes. On the other hand, the distances between the locations of the features of the acf are less sensitive to the actual distribution of the island sizes and fairly well corresponds to their mean diameter $D_{m}$. Therefore, we used the distance between the first and second feature of the acf's for our discussion.

Two examples will be presented: The topographies of polycrystalline $\mathrm{Au}$ - and Ni-films. From other methods, these materials are known to exhibit considerably different surface features. Thus they are very proper for our purpose:

Typical STM images of 20-nm thick $\mathrm{Au}$ and $\mathrm{Ni}$ films are shown in Figs. 2(a) and 2(b), respectively. Both examples are highly resolved, i.e., the deconvolution of the real surface ${ }^{10}$ gives an amount of black holes smaller than $1 \%$. The surfaces are strongly featured, showing roughnesses in the $\mathrm{nm}$ range. Clearly, the Au film exhibits a larger roughness. Figure 3 shows the roughness distributions for the two films of Fig. 2. Both distributions are shaped approximately gaussian. Due to the flat islands, however, the distribution is not perfectly, symmetric. As recognized from Fig. 2, the roughness of the Au is larger than that of the Ni surface. The half-width of $\mathbf{r}(h) d h$ found on different locations on the same sample typically amounts to $h_{1 / 2}^{\mathrm{Au}}=(7 \pm 2) \mathrm{nm}$, whereas $h_{1 / 2}^{\mathrm{Ni}}=(3 \pm 0.5)$
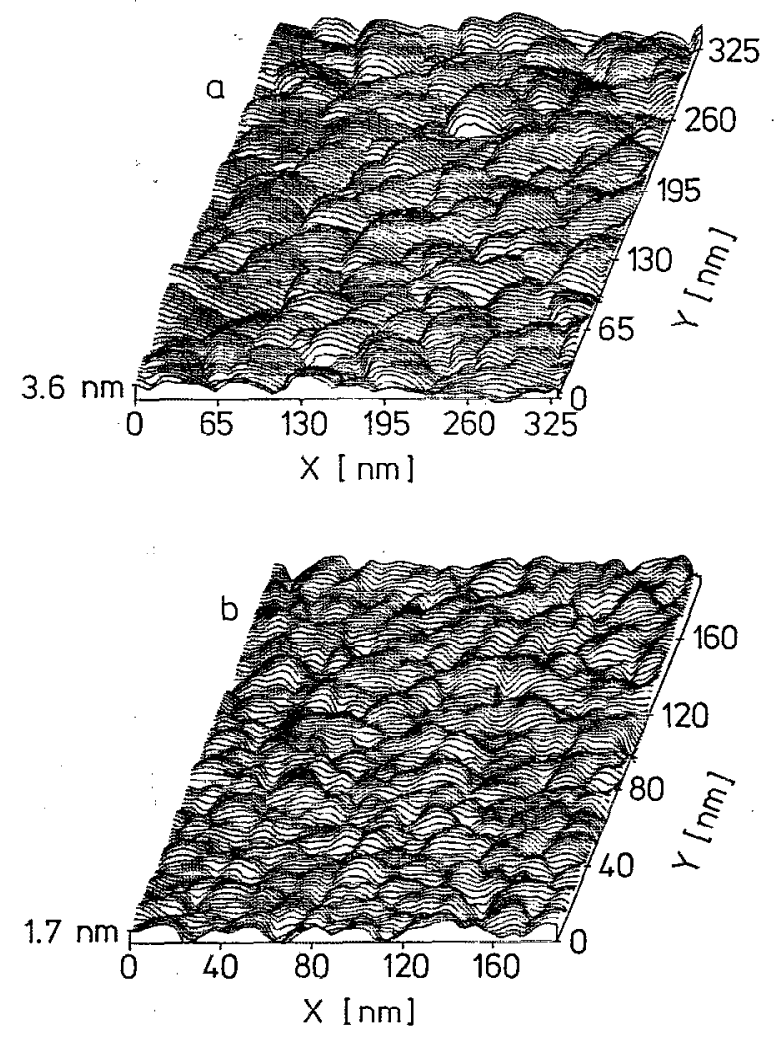

FIG. 2. (a) STM topography of a 20-nm-thick Au film evaporated at $10^{-8}$ mbar on fire polished Corning glass at room temperature. The evaporation rate was $0.1 \mathrm{~nm} / \mathrm{s}$. (b) STM topography of a Ni film prepared under the same conditions as the film in (a).

nm. Using different tips, these values remained stable provided the "black-hole criterion" concerning the resolution was satisfied.

A check of these results can be performed by transmission electron microscopy (TEM) replica techniques. This method, however, is restricted to roughnesses exceeding (4-5) $\mathrm{nm}$. For Au evaporated on glass at room temperature, the TEM values range between 8 and $12 \mathrm{~nm}^{19-21}$ in

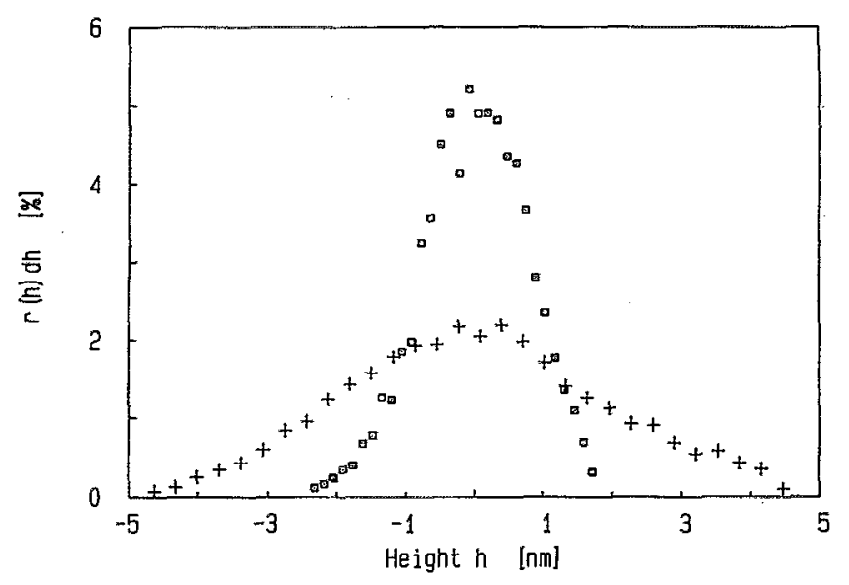

FIG. 3. The distribution of the roughness $\mathbf{r}(h) d h$ for the two films of Fig. 2. Crosses: Au film, squares: Ni film. 


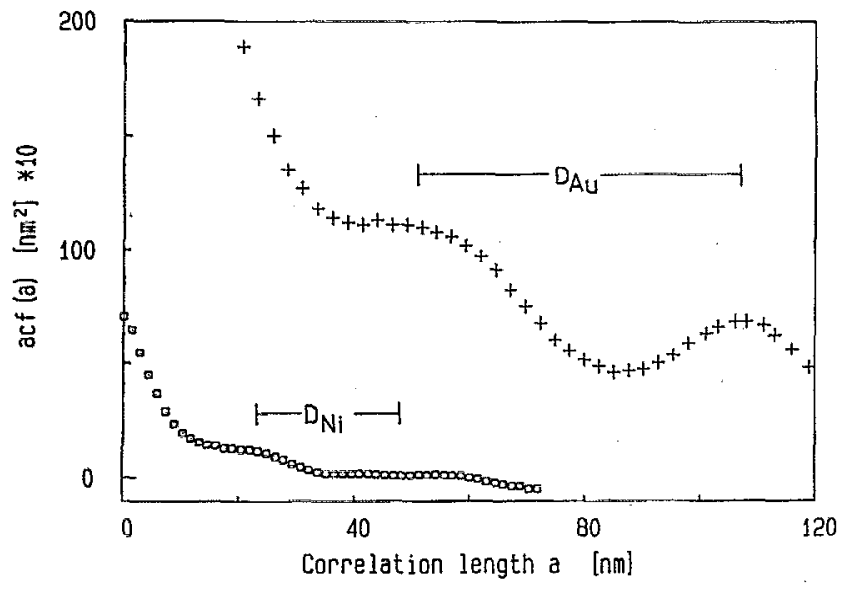

FIG. 4. Autocorrelation functions acf(a) for the two films of Fig. 2. Crosses: Au film, squares: $\mathrm{Ni}$ film. The mean island sizes evaluated from these functions are indicated.

agreement with our STM analysis. Due to the reasons mentioned before, this check was not possible for the Ni surface.

Another method, however, which additionally shows the relevance of the roughnesses for physical properties, is the measurement of the resistance during the growth of the films (see Refs. 5, 6, 21 and references therein): Usually, the thickness $d_{\text {on }}$ at the onset of ohmic conductivity of the growing film was supposed to equal approximately the mean roughness of continuous, thick samples. For $\mathrm{Au}$ on glass, typically $d_{\text {on }}=(8 \pm 3) \mathrm{nm}$ is found at room temperature. ${ }^{21}$ This value agrees with TEM replicas of thick films and additionally corresponds to the mean STM roughness evaluated in this communication. For Ni films, typical values are $d_{\mathrm{on}}=(3 \pm 1) \mathrm{nm}^{22}$ again in reasonable agreement with the STM results.

Due to the stronger supression of vertical features by blunt tips compared to lateral ones, ${ }^{9}$ the resolution is not as crucial for the evaluation of lateral sizes as for the roughnesses. The method discussed before thus can provide reasonable results even in the case of poor resolution as long as individual islands can be distinguished. For our examples, the acf's were isotropic, i.e., did not depend on the direction of a. This indicates both isotropic tip shape and film growth. Figure 4, therefore, shows the acf $(a)$ of Figs. 2 (a) and $2(\mathrm{~b})$ in dependence of $a=\sqrt{\mathbf{a}_{x}^{2}+\mathbf{a}_{y}^{2}}$. Superimposed on the usual exponential behavior, both functions show additional features produced by the distribution of the island sizes. For the discussed examples, the resulting values of $D_{m}$ again are larger for the $\mathrm{Au}$ than for the $\mathrm{Ni}$ film. The quantitative analysis yields correlation lengths of $D_{\mathrm{Ni}}=(24 \pm 3) \mathrm{nm}$ and $D_{\mathrm{Au}}=(52 \pm 3) \mathrm{nm}$. Here, the errors have been taken from acf's obtained from different locations on the same sample. Due to the reasons mentioned before, these values can be estimated using only moderately resolving tunneling tips. The features of the autocorrelations, however, are increasingly supressed for decreasing resolution.

A direct check of these results again can be performed using TEM measurements from the same films. The evaluation yields mean crystallite sizes of $D_{\mathrm{N} i}^{\mathrm{TEM}}=(25 \pm 3) \mathrm{nm}$ and $D_{\mathrm{Au}}^{\mathrm{TEM}}=(48 \pm 4) \mathrm{nm}$. Again, good agreement with the STM results can be found.

In summary, methods for a structural STM surface analysis have been presented. In order to obtain comparable results, the STM images can be quantitatively evaluated by two functions: The island sizes, i.e., the lateral extension of the corrugations, can be found using the autocorrelations of the images. Additionally, the height distributions characterize vertical features of the surface, which cannot be investigated in detail by other methods.

The mean island sizes obtained from STM agree very well with corresponding TEM results. Thus, for metallic, weakly oxidizing materials, STM can replace in part more complicated TEM investigations.

The roughness distribution describes the relative part of the surface located at a certain height. Provided the STM image is highly resolved, these STM results correspond very well with the mean roughness obtained by other methods. Concerning the investigation of, for example, thickness dependent thin-film properties, this function can provide a better understanding than mean values. Concerning the influence of surface features on the thickness dependence of the resistivity, this will be discussed elsewhere. $^{23}$

We are indebted to $\mathbf{H}$. Hoffmann for his grateful support of this work, to F. Schneider for STM software and to J. Zweck for supplying TEM images.

${ }^{1}$ K. Fuchs, Proc. Cambridge Philos. Soc. 34, 100 (1938).

${ }^{2}$ S. B. Soffer, J. Appl. Phys. 38, 1710 (1967).

${ }^{3}$ Z. Tešanović, M. V. Jarić, and S. Maekawa, Phys. Rev. Lett. 57, 2760 (1986).

${ }^{4}$ Y. Namba, Jpn. J. Appl. Phys. 9, 1326 (1970).

${ }^{5} \mathrm{~J}$. Vancea, G. Reiss, and H. Hoffmann, Phys. Rev. B 35, 6435 (1987).

${ }^{6}$ G. Reiss, K. Kapfberger, G. Meier, J. Vancea, and H. Hoffmann J. Phys.: Condens. Matter 1, 1275 (1989).

${ }^{7}$ N. Trivedi and N. W. Ashcroft, Phys. Rev. B 38, 12298 (1988).

${ }^{8} \mathrm{~J}$. Vancea, G. Reiss, F. Schneider, K. Bauer, and H. Hoffmann, Surf. Sci. 218, 108 (1989).

${ }^{9}$ G. Reiss, J. Vancea, H. Wittmann, J. Zweck, and H. Hoffmann, J. Appl. Phys. 67, 1156 (1990):

${ }^{10}$ G. Reiss, F. Schneider, J. Vancea, and H. Hoffmann, Appl. Phys. Lett. 57, 867 (1990).

${ }^{11}$ J. K. Gimzewski, A. Humbert, J. G. Bednorz, and B. Reihl, Phys. Rev. Lett. 55, 951 (1985).

${ }^{12}$ A. Bartolomé, R. Garcia, L. Vásquez, and A. M. Baró, J. Microsc. 152, 205 (1988).

${ }^{13} \mathrm{Ph}$. Niedermann and $\emptyset$. Fischer, J. Microsc. 152, 93 (1988).

${ }^{14} \mathrm{M}$. Stedman, Int. Soc. Opt. Eng. 1009, 56 (1989).

${ }^{15}$ J. P. Pelz and R. H. Koch, Phys. Rev. B 41, 1212 (1990).

${ }^{16}$ Y. Akama, E. Nishimura, A. Sakai, and H. Murakami, J. Vac. Sci. Technol. A 8, 429 (1990).

${ }^{17}$ D. K. Biegelsen, F. A. Poull, J. C. Tramontana, and S. M. Koch, Appl. Phys. Lett. 50, 696 (1987).

${ }^{18}$ J. Garnaes, F. Kragh, K. A. Morch, and A. R. Thölén, J. Vac. Sci. Technol. A 8, 441 (1990).

${ }^{19}$ L. Reimer, Elektronenmikroskopische Untersuchungs-und Präparationsmethoden (Springer, Berlin, 1959).

${ }^{20}$ R. Suhrmann, R. Gerdes, and G. Wedler, Z. Naturforsch. 18a, 1211 (1963).

${ }^{21}$ J. Vancea, Ph.D. dissertation, University of Regensburg. 1982.

${ }^{22}$ E. Hastreiter, diploma thesis, University of Regensburg, 1990.

${ }^{23} \mathrm{H}$. Brückl and G. Reiss (unpublished). 